\title{
HeloVis: a Helical Visualization for SIGINT Analysis Using 3D Immersion
}

\author{
Alma Cantu* \\ IMT Atlantique \\ Lab-STICC, CNRS \\ Thales Airborne Systems
}

\author{
Thierry Duval ${ }^{\dagger}$ \\ IMT Atlantique \\ Lab-STICC, CNRS
}

\author{
Olivier Grisvard" \\ IMT Atlantique \\ Lab-STICC, CNRS \\ Thales Airborne Systems
}

\author{
Gilles Coppin ${ }^{\S}$ \\ IMT Atlantique \\ Lab-STICC, CNRS
}

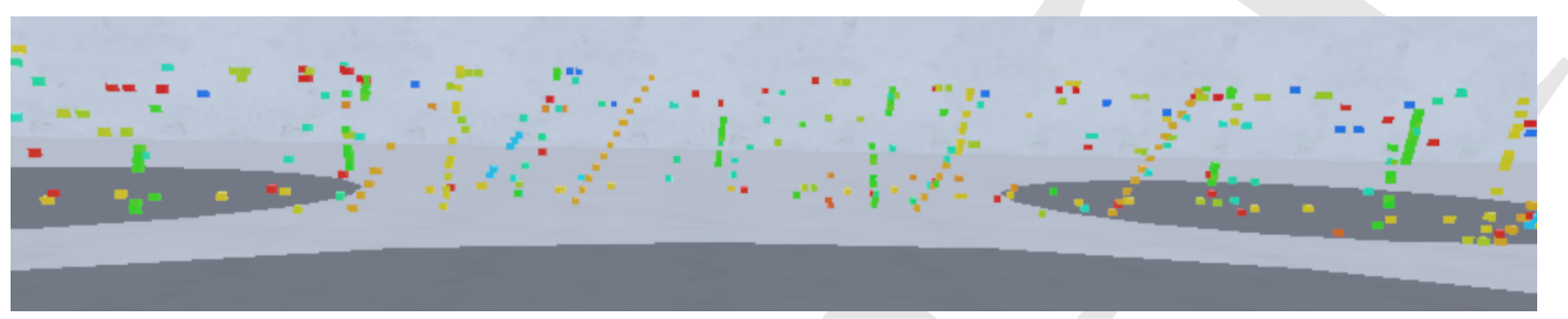

Figure 1: Alignments that indicate the presence of radar signals colored by frequency values

\begin{abstract}
In this paper we present HeloVis: a 3D interactive visualization that relies on immersive properties to improve the user performance during SIGINT analysis. SIGINT, which stands for SIGnal INTelligence, is a field facing many challenges like huge amounts of data, complex data and novice users. HeloVis draws on perceptive biases, highlighted by Gestalt laws, and on depth perception to enhance the recurrence properties contained into the data and to abstract from interferences such as noise or missing data. In this paper, we first present SIGINT and the challenges that it brings to visual analytics. Then, we present the existing work that is currently used in or that fits the SIGINT context. Finally, we present HeloVis, an innovative application on an immersive context that allows performing SIGINT analysis and we present its evaluation performed with military operators who are the end-users of SIGINT analysis.
\end{abstract}

Index Terms: Human-centered computing-Visualization-Visualization application domains-Visual analytics; Human-centered computing-Visualization-Visualization design and evaluation methods

\section{INTRODUCTION}

During a military operation, possessing knowledge about surrounding systems is primordial for the safety of the operators. Gathering this knowledge based on radar signal analysis is the objective of SIGnal INTelligence (SIGINT) operators. However, dealing with complex data such as radar signals is not the only issue that SIGINT faces so that few are the systems that fulfill the SIGINT needs. With the actual increasing amount of data these few solutions are no longer allowing a high-quality analysis on a day-to-day basis. To overcome this issue in terms of information visualization, we explore the use of 3D immersive techniques to deal with the numerous aspects of SIGINT, as suggested by Brath about the intake of 3D for visualization [2]. From this exploration we build HeloVis, an interactive visualization that makes profit of these techniques to

*e-mail: alma.cantu@imt-atlantique.fr

†e-mail: thierry.duval@imt-atlantique.fr

¥e-mail: olivier.grisvard@imt-atlantique.fr

$\S$ e-mail: gilles.coppin@imt-atlantique.fr better support SIGINT analysis. In this paper we first present the specificities of SIGINT identified thanks to interviews of military operators (in section 2). In section 3 we present the existing tools and why they do not entirely fulfill the SIGINT needs. In section 4 we present the HeloVis tool and finally, in section 5 we assess the benefits of such an approach by presenting preliminary results of an evaluation measuring user performance.

\section{SIGINT}

This section presents SIGINT through a description of its background, its data and its needs. All the following knowledge has been collected through interviews and observations of SIGINT operators.

\subsection{Background}

Radar detection consists in sending an electromagnetic emission that is reflected by objects and then intercepted back by the radar. The way the signal is distorted when reflected gives information on the objects such as distance or size. To increase the detection, the radar signals have complex characteristics that make their signature unique. Such emissions can be intercepted by other sensors named electronic support measures. Electronic Warfare (EW) exploits the interception of radar emissions to identify their origin, to characterize their threat level and to enable the appropriate reaction in case of conflict, or conversely, to prevent an opponent from doing the same. However, the identification of a radar signal requires having a precise knowledge of the existing radar signal signatures. Gathering this knowledge is an activity generally performed by dedicated military forces called SIGINT. It consists in capturing and analyzing as many emissions as possible in order to characterize their signatures and therefore fill the reference database used in electronic warfare.

\subsection{Data}

Radar signals consist of series of pulses of a certain duration spaced in time. These pulses are emitted on a specific frequency (such as radio emissions) and with a specific power, named level. Thus, pulse width $(\mathrm{PW})$, pulse repetition period (PRP), frequency and level are the four main attributes that characterize a pulse (see Fig. 2-a). These attributes may vary from one pulse to another in a same signal to increase detection or to hinder the identification. This variation is what makes the signal specific enough that it can be used for identification. Hence, the variation of the attributes is what the SIGINT operators look for but not directly what the sensor gets. Between the emission and the interception, pulses are subject to loss, 
distortion and noise, whether it be from diffraction, emitter issues or sensor issues. The sensors are able to record pulses according to their frequency, $P W$, time of arrival (TOA) and level values. In order to access the PRP, they also contain the computed delta of TOA (DTOA) such as the SIGINT datasets contain these five attributes (see Fig. 2b). Concerning the data volume, as the capacities of current radars are confidential, we cannot detail this matter but if we refer to studies made a few years ago [21] and if we consider the increase of data storage capacities, we can state that the current data volume is bigger than the number of perceptible objects that is commonly defined by the number of pixels provided by the visualization device [10].

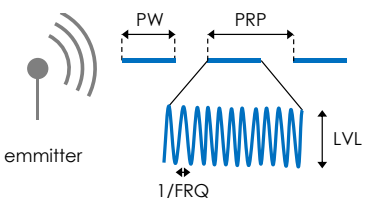

(a) emitted data

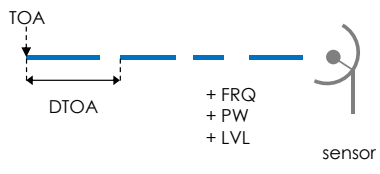

(b) intercepted data
Figure 2: Attributes of the emitted (a) and intercepted (b) data

\subsection{Needs}

To be able to identify the characteristics of a radar signal, the operators need to distinguish pulses of a same signal from noise or other signals. However, if the complexity of the variation is too high (and that happens more and more often) it is difficult to associate pulses of a same signal. A decade ago, most of the radars emitted their pulses on the same frequency such that the association was made on the frequency attribute and that the other attributes were used only to distinguish two radars with close frequency values. However the latest radars can emit on different frequency ranges creating signals with a frequency varying pseudo-randomly. To overcome this new issue the operators rely on the variation of the PW, the PRP and the level. Constrained by the need of comparison between emitted and reflected pulses, PWs and PRPs vary according to a recurrent pattern which implies similar values but also recurrent time values which make the association possible. However, the length of the pattern tends to increase more and more (to hinder identifications). Regarding the level, the emitter often rotates to cover every angle such as the intercepted level takes the shape of a set of curves which is singular enough to be detected and associated, however the emitter can also make the level vary (for depth detection purposes), which complicates the association. Consequently, to associate pulses from a same radar signal relies on identifying and correlating similar values, singular variations or recurrent time values under constraints of multidimensional data and huge amounts of data.

\subsection{Synthesis}

SIGINT deals with multi-attribute data in a huge amount that contain strong temporal aspects. Military operators have to associate the different radar signals of these datasets and identify the characteristics of the signals. In this paper we focus on the association part.

\section{RELATED WORK}

To perform the association of pulses that belong to a same signal is possible thanks to processing. By combining clustering and modulo detection, the latest algorithms allow the association of more than $80 \%$ of the SIGINT data [21]. The remaining $20 \%$, which are still in a huge amount, are unprocessed because of two issues. The first one is to be put on the account of the quality of the datasets. Despite the current algorithms being very effective, if there are too many missing data or if the noise is too important, they fail associating radar signals. The second one comes from the complexity of the radar signal. People that create radars have in mind the fact that they may be intercepted and, as such, complicate their signature to avoid their identification. Despite all the efforts to create adaptive processes, there is still a small amount of signal that is too complex to be identified automatically.

The alternative to associate the $20 \%$ radar signals remaining from processing is to rely on human perception by using representation tools. Humans have the ability to correlate despite noise or missing values and have the mind plasticity to overcome novelty [19]. Accessing the attribute values to perform the association (identifying similar values, singular variations and recurrent time values) requires their encoding on visual variables (positions, color, size, shape, etc.) [1]. According to the Gestalt law of proximity, the position permits to associate data into clusters [13]. Among the representation tools that use the position, we distinguish those that use it once, twice or three times.

Position in two dimensions is currently used by SIGINT operators aggregated with the use of multiple views to handle the multidimensional aspect (see Fig. 3). 2D charts make possible to identify similar values on the two attributes represented and can be correlated to other views with the use of color (ex: [18]). Regarding the singular variation of the level, a chart representing level over time will allow such detection. However, these charts are subject to noise and occlusion, which is complicating the selection needed for the characterization step. Recent selection tools make possible a very flexible selection [9], but because of the amount of data, providing the precision necessary to the characterization requires too much time. In terms of identification of recurrent time values, the 2D chart is of no specific support and the amount of data prevents any one-by-one pulse detection. In addition, in case of noise or multiple listening, the DTOA is irrelevant and requires to be recomputed after a signal association to provide any insight.

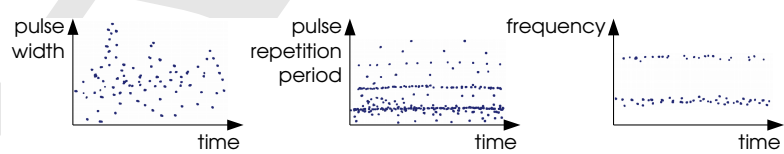

Figure 3: Illustration of the existing tools used in SIGINT analysis

The equivalent of the multiple views of 2D charts in terms of position in one dimension would be the parallel coordinates. It consists of parallel axes where each datum is represented by a line evolving over the axes (ex: [12]). This representation for SIGINT association permits to easily identify similar values on an attribute and correlate it with others. However, this representation is also subject to noise and does not allow the access to neither the level variation nor the recurrent time values.

Concerning the use of position in three dimensions, it is implemented by $3 \mathrm{D}$ charts. 3D charts extend the correlation on a row to three attributes or more if we consider other visual variables [6]. Concerning its use, some recent studies have proven cluster detection to be more effective on stereoscopic displays [8], implying the use of stereoscopic screens or immersive devices. But 3D representations still face occlusion and distortion issues that call their use into question [16].

Finally, the SIGINT data can also be represented according to other visual attributes. Using the color may correspond to the use of a pixel view which consists in representing data by pixel size squares, coloring them according to their values and displaying them side by side (ex: [15]). This representation, that is exempt from occlusion, can allow displaying multidimensional data by representing each datum by a stack, each square representing an attribute. Concerning the selection, as the attributes are encoded by color but not by position, data with similar values are not necessarily displayed next to another, disabling the possibility to spatially select data with similar values. However, there exist some selection tools according 
to other visual variables such as color filters (ex: [4]), but as for the spatial selection, these tools face noise, issues to select level variation and difficulties to identify temporal recurrences.

As such, there is no solution that completely fulfills the need of the SIGINT field by handling in a same time singular variations and recurrent time values under the constraints of huge amounts of data, complex data and complex tasks.

\section{Proposition}

We have identified that the main issue is brought by biases among the data such that we decide to base our analysis only on the time value that is less subject to error and that contains, through its recurrence, the information of belonging to the signal.

\subsection{HeloVis principle}

HeloVis consists in an interactive $3 \mathrm{D}$ visualization within which each pulse is represented by an object positioned on an helical scale depending on its time value. As such, each object is positioned according to the following formula:

$$
(y, r, \theta)=\left\{\begin{array}{l}
t \\
R \\
2 \pi(t \bmod p)
\end{array}\right.
$$

where $(y, r, \theta)$ is the position of the object in a cylindric referential, $t$ the time value, $R$ a constant defining the size of the representation, and $p$ (for period) a variable influencing the helical twist. The user can smoothly modify the value of $p$ impacting the helical scale by twisting or untwisting it. Potentially the user will reach a value of $p$ that corresponds to the PRP of a radar signal that $s /$ he will be able to detect because the pulses of this radar will be aligned (see Fig. 4).

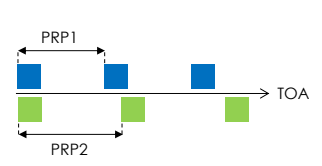

(a) linear time

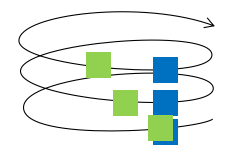

(b) helical time, with pe- (c) helical time, with period $=$ PRP1

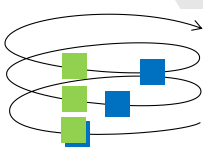

riod $=$ PRP2
Figure 4: Different representations of two different radar signals

In case of several PRP on a same signal, several alignments will appear over the cylinder (see Fig. 5). Concerning missing values, they will correspond to a hole in the detected alignment, which will not impact the detection according to the Gestalt law of continuity [13]. Finally, a radar signal does not necessarily have a constant PRP value such that the detection will not necessarily be an alignment but at least a geometric shape, as PRP variations are always defined by functions.

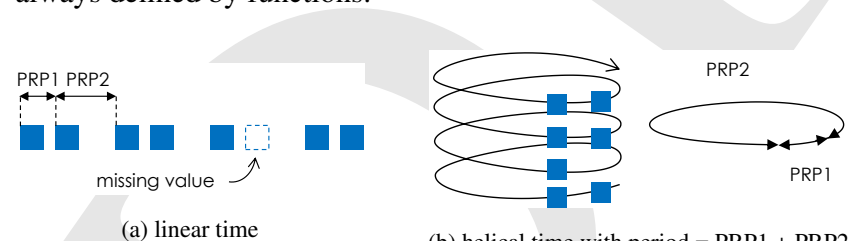

Figure 5: Different representations of a radar signal with several repeated pulses and missing values

\subsection{Other features}

To be able to conduct the SIGINT analysis on such an interactive visualization, we add other features. The first one is the possibility to use the color to encode another attribute. That may be the frequency, the level or the pulse width. Such encoding allows us to consolidate the detection and quickly acquire knowledge over the distinguished signals. We also propose to encode an attribute on another dimension, the radius (see Fig. 6). This makes possible, as for the color, to consolidate the detection but also to enhance the selection thanks to the spatial distribution. We also add some interaction tools allowing a direct access to the pulses, one displaying the values of each pulse and one calculating the DTOA among two selected pulses. We also add a selection tool to isolate the detection on another view, which permits to record an association and analyze it without other surrounding pulses. Finally, to facilitate the interaction and increase the user experience, we add some features specific to the immersive environment such as a teleportation tool.

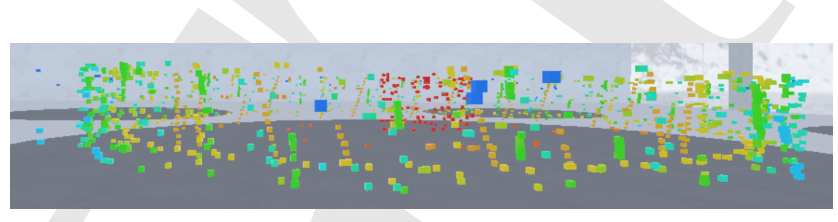

Figure 6: View encoding frequency by color and by radius position on HeloVis

\subsection{D visualization}

Although the choice of a 3D visualization seems implicit to the helical visualization metaphor, the use of $3 \mathrm{D}$ is questionable as its contribution does not necessarily compensate for its many drawbacks such as occlusion or the loss of information brought by perspective distortion [16]. Representation of 3D spatial data is one of the few fields where the benefits of using 3D visualization outweigh the disadvantages and only for shape perception tasks [16]. Thus, regarding the representation of abstract data, $3 \mathrm{D}$ visualization is often considered as unnecessary [16] or even ineffective [20]. Whether these criticisms concern the use of flattened 3D or the use of 3D with depth perception (stereoscopic and motion parallax), these two methods are to be distinguished. Indeed, numerous studies comparing flattened 3D and 3D with depth have established significant performance differences [2]. This does not mean that the 3D with depth overcomes all the disadvantages of 3D but it can potentially improve the balance in some cases such as the one that concerns us.

In the case of the HeloVis representation, which main perceptual need is to identify shapes, 3D makes it possible to respect the metaphor of representation, which is a helical coordinate system, but also to encode an additional attribute. Adding this attribute requires identifying three-dimensional shapes that is done best using 3D with depth [8]. In parallel, here we propose to use immersive $3 \mathrm{D}$, which increases the display area and therefore the management of large amounts of data. Regarding the disadvantages, the problems of occlusion and loss of information related to the distortion are still present. However, according to the Gestalt law of the good form, a standard geometric shape, even covered by another, will always be distinguished as a unique form, which is the case of the clusters highlighted by HeloVis. With regard to the loss of information, HeloVis is meant to identify forms so the distortion of the information is not a disadvantage. Some studies state that if $3 \mathrm{D}$ visualization does not provide a better precision judgment, it facilitates shape understanding $[5,11]$. Thus, because of its needs, its contributions and also its disadvantages, HeloVis is a tool where the use of a 3D with depth visualization is relevant.

\subsection{Existing helical representations}

The use of a helical baseline to highlight time recursions already exists in other contexts. The most relevant work is a helical representation to handle geographic dataset [7]. If this technique is also interactive, allowing us to identify recursions based on any period, it differs from our proposition in a manner that makes it unusable for 
SIGINT. First of all, it does not permit to access the same amount of data because it is meant to be part of a graphic panel and as such is constrained by the size of the display. HeloVis being an immersive tool permits an infinite extension of the display size. Moreover, this existing work permits to access the data from outside the cylinder which raises occlusion issues and the width of the cylinder is not large enough to render the complexity of SIGINT data.

Another way to handle time recursions is by using spiral representations which can be considered as helicoids distorted by perspective. Tominski and Schumann present an interactive spiral for spotting patterns in time series data [17]. The use of a spiral permits to place the user inside the data and as such avoid occlusion issues but this flatten helicoid raises distortion issues that, if they do not hinder the pattern detection, can lead to misperceive the correct alignment period. In addition, as for many spiral representations, the work of Tominski and Schumann is meant for continuous time values, which is not the case of SIGINT data and which compromises the use of the perceptive continuity law to detect patterns.

\section{Evaluation}

We believe that HeloVis allows the users to perform a better association. To challenge this assumption we conducted an exploratory evaluation comparing the user performances between the tool currently used by SIGINT operators and HeloVis. A better association implies a better association rate and a better quality of the associations such that we make the following hypothesis:

H1 - The quantity of the radar signals associated is improved by the use of HeloVis compared to the reference tool.

$\mathrm{H} 2$ - The quality of the radar signals associated is improved by the use of HeloVis compared to the reference tool.

\subsection{Experimental framework}

As this evaluation falls into the category of user performance evaluation of Lam et al., we have designed it to access objective metrics related to our hypothesis [14]. As an exploratory evaluation we did not want it to be biased by the usability such that we did not collect time metrics or error rate but only metrics about the quantity and quality of the associations made by the participants. In addition, to master the experimental conditions of the evaluation we recorded demographic data and data related metrics. And finally, to be able to get an insight about the acceptability of HeloVis, we recorded subjectives metrics about user experience.

\subsubsection{Apparatus}

The evaluation was done on two different devices: a standard computer on which the reference system was used, and a more powerful laptop, coupled with an immersive helmet to use HeloVis. The standard computer was composed of a central unit, a mouse, a keyboard and a 24 -inch screen with a resolution of $1920 \times 1080$ pixels. The main unit had $8 \mathrm{~GB}$ of RAM, an Intel Core i7 processor and used the operating system Windows 7 (64-bit). The laptop has been equipped with an immersive helmet HTC Vive, with two controllers and two position sensors. It had $32 \mathrm{~GB}$ of RAM, an Intel Core $i 7$ processor, a Nvidia $980 M$ graphics card, and the operating system Windows 8 (64-bit). The immersive headset had a resolution of $2160 \times 1200$ pixels that provided a horizontal field of view of 110 degrees.

\subsubsection{Participants}

We recruited eleven participants aged between 34 and 52 to perform our evaluation. Among the participants the females were underrepresented (1/11) but this reflects the reality of SIGINT. Half of the participants were military people (5/11) and half of them were civilian engineers building military solutions (6/11). Most of them worked in the field of SIGINT (8/11) and the remaining ones worked in the field of electronic warfare which is related to SIGINT. Concerning their level of expertise, half of them were full experts, able to associate even the most complex radar signals (6/11) and half of them had complete knowledge and understanding of the field but not the experience to associate all radar signals (5/11), irrespective of their profession. Finally, only few of them had previous experience with immersive devices (3/11). These participants have the knowledge and the experience to perform a SIGINT analysis and as such, are representative of the targeted users of HeloVis.

\subsubsection{Procedure}

After following a generic experimentation procedure (to ask to sign consent and to get demographic metrics), we asked them to perform a simple task on two different systems: HeloVis and a reference system that is currently used by SIGINT operators. The first system that they had to use was different from one consecutive participant to another in order to remove biases. The reference tool, whom we cannot talk too much about because of confidential restriction, was a set of 2D charts displaying impulsions over different attributes (see Fig. 3). The task was to associate as much radar signal as they could with the best quality. Finally we asked them to fulfill a SUS questionnaire [3] to record their insight. From this procedure we obtained demographic data and subjective metrics and extracted objective metrics from the logs of the associations. The number of associations gave us information about $\mathrm{H} 1$ and the pulses contained in each association gave us information about $\mathrm{H} 2$.

\subsubsection{Synthesis}

During the evaluation, we asked SIGINT experts to perform a same association task on two different systems, the system currently used by SIGINT operators on a desktop and HeloVis on an immersive device. This evaluation provided us with objectives metrics to confront our hypothesis and subjectives metrics to give us insight on user experience.

\subsection{Results}

Thanks to the extracted metrics we got insight on the quantity and the quality of the association. We extracted this from the recorded metrics considering the participants and the radar signals as independent variables and considering the number of selections, the noise percentage and the selection percentage as dependent variables.

\subsubsection{Quantity of the association}

To validate $\mathrm{H} 1$ we compared the quantity of associations performed on each system. Among 88 possible selections (11 participants x 8 radar signals) the participants associated 53 signals with HeloVis and 43 with the reference tool. This result highlights the fact that HeloVis makes possible a better performance in terms of quantity, validating $\mathrm{H} 1$. In addition, this highlights the fact that the number of measurements is not identical for both systems. To include this variable in the rest of the analysis, in particular in the analysis of the quality, requires to identify the nature of the relation between the quantity and quality that we are not able to provide. We therefore establish a postulate of independence between quantity and quality in order to be able to continue the analysis. From this point, we consider that HeloVis and the reference tool are two different samples and do not take anymore into account the number of associations.

\subsubsection{Quality of the association}

To validate $\mathrm{H} 2$ we compare the reference tool and HeloVis based on the percentage of noise of each association and the percentage of selection (see Table 1). From this analysis we observe that the percentage of noise is lower for HeloVis and that the percentage of selection is higher for HeloVis. The percentage of noise selected points out the usability of the selection. A selection with too much noise can prohibit any use of processing starting with the use of the DTOA and can lead to misunderstand many characteristics of the radar. The percentage of signal selected indicates the quality 
of knowledge about the selected signal. The more important the value is, the more the signal characteristics are known and less noise stays in the dataset. Regarding the meaning behind the metrics of noise and selection, HeloVis seems to permit a better quality of the selection. We extract this value thanks to a Wilcoxon test as the amount of data does not allow us to assume that it follows a normal distribution and as the data fail the normality test, invalidating the use of the Student test. From this result we can conclude that using HeloVis improves the quality of the selection in terms of noise percentage and selection percentage, validating $\mathrm{H} 2$.

\begin{tabular}{l|l|l} 
& selection & noise \\
\hline reference tool & $83.4 \% \pm 8.5 \%$ & $27.8 \% \pm 9.2 \%$ \\
\hline HeloVis & $98.5 \% \pm 0.9 \%$ & $0.4 \% \pm 0.3 \%$ \\
\hline p value & $2.064 \mathrm{e}-6$ & 0.0114
\end{tabular}

Table 1: Result of the Wilcoxon test on the selection and noise percentage by associations on HeloVis and on the reference tool.

\subsubsection{Impact of system order}

To identify the impact of using the same set of data when evaluating the two systems, we compare the quality of the detections according to the order in which the tools were used (see Table 2). According to the selection percentage and the noise percentage, there are no major differences between the results with HeloVis and the reference tool depending on the order of use. Regarding the differences within the same tool we find that the selection is better for the first tool used which is counterintuitive. Regarding the noise percentage it decreases for the second use as we could expect. These internal variations, however, have no impact as long as they are equivalent on each tool, given that the order has been changed in a homogeneous manner.

\begin{tabular}{l|l|l|l|} 
& order & selection & noise \\
\hline reference tool & 1 & $83.9 \% \pm 8.5 \%$ & $28.9 \% \pm 8.9 \%$ \\
\hline reference tool & 2 & $82,7 \% \pm 8.5 \%$ & $26.3 \% \pm 9.4 \%$ \\
\hline HeloVis & 1 & $98.6 \% \pm 0.8 \%$ & $0.5 \% \pm 0.4 \%$ \\
\hline HeloVis & 2 & $98.2 \% \pm 1 \%$ & $0.2 \% \pm 0.2 \%$
\end{tabular}

Table 2: Selection percentage and noise percentage on HeloVis and the reference tool according to the order of use.

\subsubsection{Subjective results}

During the evaluation, we have recorded subjectives metrics by noting users' comments and asking them to fulfill a SUS questionnaire [3]. The reference tool was considered as badly designed in a way that complicates the interaction. This comforts us in our decision not to base performance metrics on usability, but only on perception as we did by measuring metrics on the signal detections. In contrary, HeloVis was described as very easy to use. The synthesis of all these comments toward usability was transcribed in the SUS questionnaires results: 50 for the reference tool and 74 for HeloVis. As we do not want to evaluate the interaction we will not take into account this result, however, it indicates that HeloVis was positively accepted by SIGINT experts who represent an important part of the SIGINT community.

\subsubsection{Summary}

To conclude, we have identified that HeloVis seems to allow a better quantity of association however we were not able to validate its significance. Concerning the quality of the association we have validated the fact that HeloVis permits to perform a better quality association. Finally, we noted from the subjective comments that the users are afraid to miss information on both systems.

\section{CONCLUSION}

The proposed tool HeloVis is designed to meet the requirements of SIGINT, which are: managing complex data, complex tasks, huge amounts of data and potentially novice users. These specificities are such that there are no existing techniques that meet them all. HeloVis relies on the Gestalt perceptive law and 3D immersive aspects to provide a new way to perform SIGINT analysis. According to an exploratory evaluation achieved with SIGINT experts, we established that HeloVis permits to perform more efficiently the first part of SIGINT analysis: the signals association. In addition, it was positively accepted by the participants in term of usability. In further studies, we aim to continue our research and perform complementary evaluations of HeloVis such as taking into account the complexity of the radar signal and the participants' expertise.

\section{REFERENCES}

[1] J. Bertin. Semiology of Graphics. ESRI Press, 2011.

[2] R. Brath. 3d infovis is here to stay: Deal with it. Proc. of the IEEE VIS International Workshop on 3DVis, pp. 25-31, 2015.

[3] J. Brooke et al. Sus-a quick and dirty usability scale. Usability evaluation in industry, 189(194):4-7, 1996.

[4] F. Chevalier, P. Dragicevic, and C. Hurter. Histomages: fully synchronized views for image editing. Proc. of the ACM symposium on User interface software and technology, pp. 281-286, 2012.

[5] G. Cliquet and al. Towards hmd-based immersive analytics. In Immersive analytics Workshop, IEEE VIS 2017, Oct. 2017.

[6] C. Donalek and al. Immersive and collaborative data visualization using virtual reality platforms. Proc. of the IEEE International Conference on Big Data Immersive, pp. 609-614, 2014.

[7] J. Gautier, P.-A. Davoine, and C. Cunty. Helical time representation to visualize return-periods of spatio-temporal events. In 19th AGILE International Conference on Geographic Information Science, 2016.

[8] N. Greffard, F. Picarougne, and P. Kuntz. Visual community detection: An evaluation of $2 \mathrm{~d}, 3 \mathrm{~d}$ perspective and $3 \mathrm{~d}$ stereoscopic displays. Graph Drawing, pp. 215-225, 2011.

[9] C. Hurter, B. Tissoires, and S. Conversy. Fromdady: Spreading aircraft trajectories across views to support iterative queries. IEEE Transactions on Visualization and Computer Graphics, 15(6):1017-1024, 2009.

[10] T. Jankun-Kelly and al. Scalability considerations for multivariate graph visualization. In Multivariate Network Visualization, pp. 207235. Springer, 2014.

[11] M. John, M. B. Cowen, H. S. Smallman, and H. M. Oonk. The use of $2 \mathrm{~d}$ and $3 \mathrm{~d}$ displays for shape understanding versus relative-position tasks. Human Factors, 43(1):79-98, 2001.

[12] D. A. Keim. Information visualization and visual data mining. IEEE Transactions on Visualization and Computer Graphics, 8(1):1-8, 2002.

[13] K. Koffka. Principles of Gestalt psychology, vol. 44. Routledge, 2013.

[14] H. Lam and al. Seven guiding scenarios for information visualization evaluation. 2011.

[15] J. Landstorfer and al. Weaving a carpet from log entries: A network security visualization built with co-creation. Proc. of the IEEE Conference on Visual Analytics Science and Technology, pp. 73-82, 2015.

[16] T. Munzner. Rules of thumb. In Visualization analysis and design. CRC Press, 2014

[17] C. Tominski and H. Schumann. Enhanced interactive spiral display. In Proc. of the Annual SIGRAD Conference, number 034, pp. 53-56. Linköping University Electronic Press, 2008.

[18] C. Viau and M. J. McGuffin. Connectedcharts: Explicit visualization of relationships between data graphics. Proc. of the Eurographics Conference on Visualization, 31(3):1285-1294, jun 2012.

[19] J. Wagemans and al. A century of gestalt psychology in visual perception: Ii. conceptual and theoretical foundations. Psychological bulletin, 138(6): 1218, 2012.

[20] C. Wickens, D. Merwin, and E. Lin. Implications of graphics enhancements for the visualization of scientific data: Dimensional integrality, stereopsis, motion, and mesh. Human Factors, 36(1):44-61, 1994.

[21] A. A. T. Zade and A. M. Pezeshk. Pulse repetition interval detection using statistical modeling. Proc. of the 8th International Conference on Signal Processing Systems, pp. 100-104, 2016. 